\title{
“Asleep" deep brain stimulation for essential tremor
}

\author{
Tsinsue Chen, MD, ${ }^{1}$ Zaman Mirzadeh, MD, PhD, ${ }^{1}$ Kristina Chapple, PhD, ${ }^{1}$ \\ Margaret Lambert, RN, BSN, ${ }^{1}$ Rohit Dhall, MD, ${ }^{2}$ and Francisco A. Ponce, MD ${ }^{1}$
} Divisions of ${ }^{1}$ Neurological Surgery and ${ }^{2}$ Neurology, Barrow Neurological Institute, St. Joseph's Hospital and Medical Center,
Phoenix, Arizona

\begin{abstract}
OBJECTIVE Deep brain stimulation (DBS) performed under general anesthesia ("asleep" DBS) has not been previously reported for essential tremor. This is in part due to the inability to visualize the target (the ventral intermediate nucleus [VIM]) on MRI. The authors evaluate the efficacy of this asleep technique in treating essential tremor by indirect VIM targeting.

METHODS The authors retrospectively reviewed consecutive cases of initial DBS for essential tremor performed by a single surgeon. DBS was performed with patients awake $(n=40$, intraoperative test stimulation without microelectrode recording) or asleep ( $n=17$, under general anesthesia). Targeting proceeded with standardized anatomical coordinates on preoperative MRI. Intraoperative CT was used for stereotactic registration and lead position confirmation. Functional outcomes were evaluated with pre- and postoperative Bain and Findley Tremor Activities of Daily Living scores.
\end{abstract}

RESULTS A total of 29 leads were placed in asleep patients, and 60 were placed in awake patients. Bain and Findley Tremor Activities of Daily Living Questionnaire scores were not significantly different preoperatively for awake versus asleep cohorts $(p=0.2)$. The percentage of postoperative improvement was not significantly different between asleep $(48.6 \%)$ and awake $(45.5 \%)$ cohorts $(p=0.35)$. Euclidean error $(\mathrm{mm})$ was higher for awake versus asleep patients $(1.7 \pm$ 0.8 vs $1.2 \pm 0.4, p=0.01)$, and radial error $(\mathrm{mm})$ trended higher for awake versus asleep patients $(1.3 \pm 0.8$ vs $0.9 \pm 0.5$, $p=0.06)$. There were no perioperative complications.

CONCLUSIONS In the authors' initial experience, asleep VIM DBS for essential tremor without intraoperative test stimulation can be performed safely and effectively.

http://thejns.org/doi/abs/10.3171/2015.6.JNS15526

KEY WORDS asleep DBS; essential tremor; intraoperative imaging; functional neurosurgery

$\mathrm{D}$ EEP brain stimulation (DBS) is typically performed with intraoperative test stimulation in awake patients. For patients with essential tremor, the target is often the ventral intermediate nucleus (VIM). Indirect targeting is achieved by placing leads at consensus coordinates based on stereotactic anatomical atlases, and repositioning as needed based on electrophysiological mapping and/or test stimulation with the latter determining clinical benefit and side-effect profile., ${ }^{2,621,30}$ There is growing interest in performing DBS on patients with Parkinson's disease under general anesthesia. ${ }^{8,32}$ This socalled "asleep" DBS technique relies on direct targeting of either the globus pallidus pars interna or subthalamic nucleus on MRI, ${ }^{13,22,24}$ coupled with intraoperative MRI or CT to determine the stereotactic accuracy of lead placement. ${ }^{1,16,19,25,26}$ Revisions are made as needed based on stereotactic error.

Asleep DBS relies on intraoperative imaging to verify the accuracy of direct targeting at the time of lead placement, effectively establishing stereotactic accuracy as the primary surgical end point. ${ }^{11,12}$ The efficacy of asleep DBS has been largely predicated on the ability to visualize the target on MRI. Applying this technique to patients with essential tremor is therefore complicated because the target VIM is not discernable on 1.5-T or 3-T MRI. Therefore, stereotactic VIM targeting uses indirect targeting meth-

ABBREVIATIONS AC-PC = anterior commissural-posterior commissural; $A D L=$ activities of daily living; $D B S=$ deep brain stimulation; $H O B=$ head of bed; $I C=$ intercommissural; $\mathrm{iCT}=$ intraoperative $\mathrm{CT}$; VIM = ventral intermediate nucleus.

SUBMITTED March 6, 2015. ACCEPTED June 4, 2015.

INCLUDE WHEN CITING Published online November 27, 2015; DOI: 10.3171/2015.6.JNS15526. 
ods. ${ }^{4,28,29}$ To assess the impact on outcomes of limitations introduced by indirectly targeting the VIM, we retrospectively reviewed DBS of the VIM on patients who underwent DBS placement under general anesthesia without intraoperative test stimulation.

\section{Methods Patients}

VIM DBS was performed in 61 consecutive patients with essential tremor between March 1, 2012, and June 30, 2014, by a single surgeon. Of these 61 patients, $41(67.2 \%)$ underwent awake DBS with intraoperative test stimulation and 20 (32.8\%) underwent asleep DBS (i.e., under general anesthesia). Patients who underwent previous surgical or radiation-based treatment for essential tremor were excluded. Final analysis included 40 awake and 17 asleep patients undergoing DBS. The decision to perform surgery under general anesthesia was based on patient preference and the referring neurologist's approval and informed consent emphasizing that awake surgery is the more accepted and established method. The advantages of awake surgery and paucity of data supporting asleep surgery were explicitly reviewed. Patients were also informed that asleep DBS does not include intraoperative test stimulation, and US FDA labeling for DBS includes a prerequisite for successful intraoperative test stimulation; therefore, asleep DBS is considered an off-label procedure.

The decision to perform unilateral versus bilateral DBS was made by a multidisciplinary consensus team (including the patient's neurologist) based on the severity of tremor disability and degree of bilateral contribution. Patients undergoing bilateral DBS were informed that the FDA has approved unilateral DBS only. They were counseled that bilateral VIM DBS surgery is off label and were informed of the potential risks. This study was approved by the St. Joseph's Hospital and Medical Center Institutional Review Board.

\section{Targeting and Surgical Procedure}

One of 2 preoperative image sequences was obtained using a 3-T MRI scanner for direct targeting: a T1-weighted magnetization-prepared rapid gradient-echo (MP RAGE) sequence (Siemens) with 1-mm slice thickness or a T1weighted spoiled gradient recalled echo (SPGR) sequence (General Electric) with 2-mm slice thickness (1-mm skip). VIM targeting proceeded with the following protocol: X coordinate, $10.5 \mathrm{~mm}$ lateral to the lateral ventricular wall and no more than $14 \mathrm{~mm}$ lateral from midline; Y coordinate, $25 \%$ of the anterior commissure to posterior commissure (AC-PC) distance posterior to the midcommissural point; and $\mathrm{Z}$ coordinate, at the AC-PC plane. The trajectory was chosen with the entry point on a gyrus, avoiding the ventricle by at least $4 \mathrm{~mm}$.

For the "asleep" technique, both frameless (NexFrame, 5 patients) and frame-based stereotaxy (Leksell, 12 patients) were used. (Our "asleep" protocol changed from frameless to frame-based placement in January 2013. At that time, we started keeping the head of bed [HOB] flat, i.e., $0^{\circ}$, for asleep DBS surgery. A recent multivariate analysis demonstrated that there was a significant improvement in accuracy and decrease in case and operative time before and after this transition. ${ }^{18}$ ) Following induction of general anesthesia, the patients had the stereotactic frame or NexFrame bone fiducials placed in the operating room. Intraoperative CT (iCT) images (1.25-mm slice thickness) were obtained using a CereTom or BodyTom mobile CT scanner (NeuroLogica) immediately after frame or bone fiducial placement, and image coregistration with the preoperative CT was completed using Medtronic Framelink Software. DBS leads were placed, and a postimplant iCT scan was obtained prior to skin closure. The stereotactic error was then measured on the postimplant scan, and if the radial error was less than $2 \mathrm{~mm}$, the skin was closed. If a lead was repositioned, an additional CT scan was obtained prior to closure to verify accuracy.

In the awake cohort, all leads were placed using the Leksell stereotactic frame. Patients were positioned with the $\mathrm{HOB}$ at $30^{\circ}$ for patient comfort and to facilitate airway patency during periods of intraoperative sedation. After the lead(s) were placed, patients underwent intraoperative test stimulation without microelectrode recording to confirm tremor resolution and lack of sustained side effects. Repositioning was performed based on absence of clinical benefit, or unacceptably low thresholds for capsular or sensory side effects. Test stimulation was repeated after new lead placement, and the higher threshold was verified and documented in the operative report. CT scans were obtained postoperatively to confirm accuracy. Intraoperative fluoroscopy was not used, and all bilateral electrodes were implanted in one operation.

Across the awake cohort, the following factors varied: where the registration CT was performed (the radiology department [ $\mathrm{n}=16$ patients] vs the operating room [n = 24]), where the stereotactic frame was placed (the preoperative area $[n=10]$ vs the operating room $[n=30]$ ), where the postimplant CT was performed (the radiology department $[n=12]$ versus the operating room $[n=28]$ ). (In our current protocol, all of these steps now take place in the operating room.)

Among 17 patients undergoing asleep DBS, 29 DBS leads were placed (12 bilateral, 5 unilateral). Among 40 patients undergoing awake DBS, 60 leads were placed (20 bilateral, 20 unilateral). The left lead was placed first in bilateral cases. The second contact from the tip of the DBS lead (i.e., Contact 1, Medtronic model 3387) was placed to target. Intraoperative test stimulation was performed at $0-4 \mathrm{~V}, 90-\mu$ sec pulse width, and $180-\mathrm{Hz}$ frequency. DBS pulse generators were placed 7-10 days after the electrode implantation in 15 asleep patients $(88 \%)$ and 34 awake patients $(85 \%) ; 2$ patients (12\%) in the asleep group and 6 patients $(15 \%)$ in the awake group had generators placed on the same day as DBS. Operating room time reflected the total duration of procedure from the time the patient was wheeled into the operating room to the time he or she was wheeled out. Case time reflected time from skin incision to completion of skin closure.

\section{Stereotactic Accuracy Assessment}

Stereotactic errors were calculated with Framelink software, based on preoperative target coordinates and trajectory and position of Contact 1 as determined on iCT (Fig. 1). Euclidean error corresponded to the magnitude of the error vector between coordinates of the principal con- 


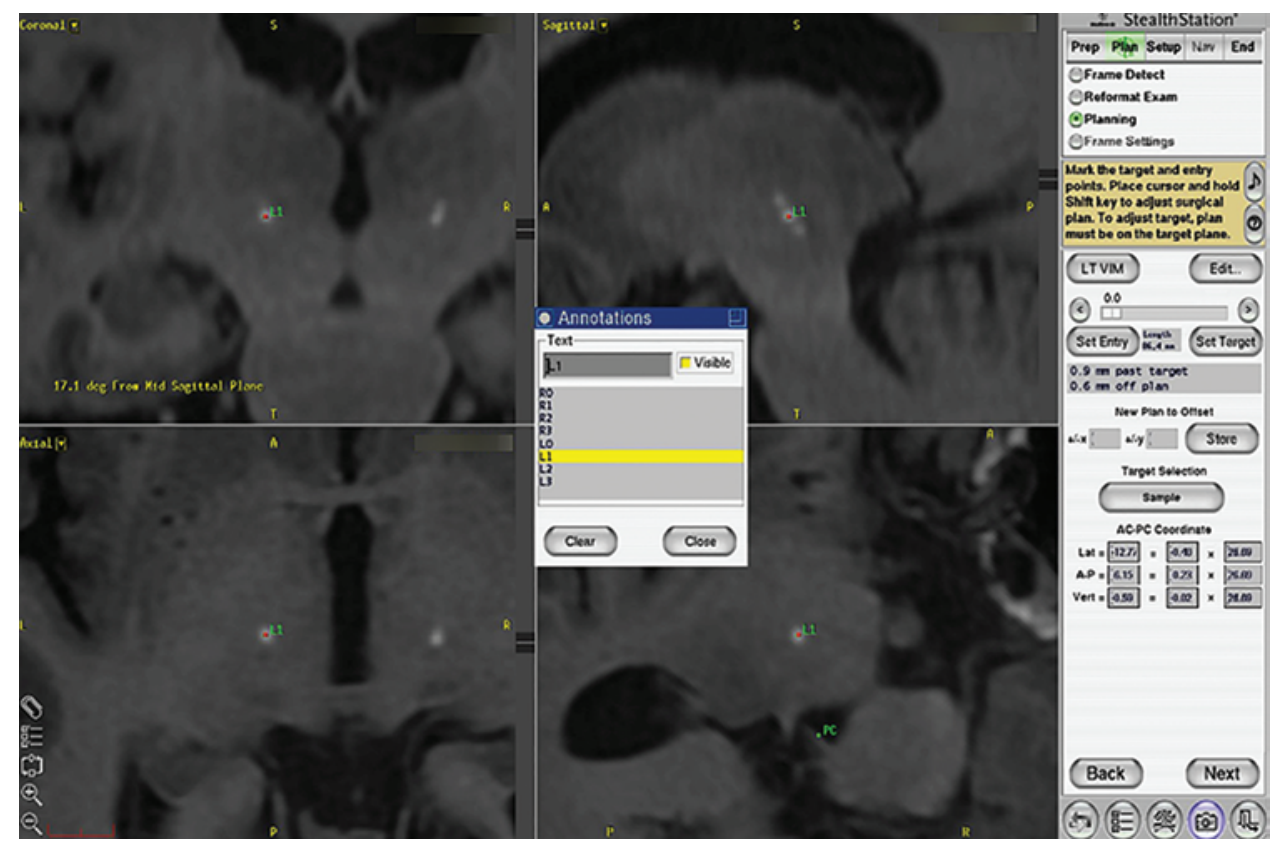

FIG. 1. Framelink image of ventral intermediate nucleus deep brain stimulation contact 1 placed to target with listed coordinates and calculated error. Figure is available in color online only.

tact and planned target. Radial error indicated the amount of deviation from the planned trajectory, or the shortest distance from Contact 1 to the planned trajectory.

\section{Functional Outcomes Assessment}

The Bain and Findley Tremor Activities of Daily Living (ADL) Questionnaire was mailed to patients postoperatively. ${ }^{3,5}$ Patients were asked to complete the questionnaire based on preoperative and postoperative self-assessment. Of the 17 patients in the asleep group, 11 (64.7\%) responded to the questionnaire; time of response ranged from 4 to 29 months after surgery (mean $17.7 \pm 7.4$ months). Of the 40 patients in the awake group, 19 (48\%) responded to the questionnaire; however, 1 patient suffered a new disability due a motor vehicle accident and was excluded from final analyses; follow-up time ranged from 4 to 31 months postoperatively (mean $16.4 \pm 8.0$ months).

An additional telephone survey was administered to 12 patients from the asleep group between 2 and 27 months postoperatively (mean $14.5 \pm 7.4$ months). The remaining patients in the asleep group were unable to be contacted by phone. This survey consisted of 4 questions: 1) Would you undergo surgery again? 2) Would you recommend asleep DBS to others? 3) Would you prefer asleep DBS if given the option? 4) Did your quality of life improve after DBS surgery?

\section{Data Analysis}

Frequencies and descriptive statistics including counts, percentages, means, and standard deviations are presented. Statistics are presented as means \pm standard deviations. Outcomes were compared with independent samples ttests. The average difference in error for left- versus rightside leads was analyzed using an independent samples t- test. A power analysis suggested that 28 patients in total were required to achieve $80 \%$ power to detect a 10-point mean difference in Bain and Findley Tremor ADL scores between the awake and asleep cohorts, based on mean scores of 60 and 50, with an SD of 10. p $<0.05$ was considered statistically significant. Data were analyzed using SPSS version 21.

\section{Results}

\section{Functional Outcomes Assessment}

All 12 patients who underwent asleep DBS responded "yes" to the 4 telephone survey questions described above. There was no statistically significant difference in Bain and Findley Tremor ADL scores in the awake versus asleep groups at baseline $(p=0.2)$ or postoperatively $(p=$ 0.66 ) (Table 1). For the asleep cohort, the average preoperative score was $66.0 \pm 13.3$, and the average postoperative score was $33.9 \pm 13.1$, a decrease of 32.1 points $(48.6 \%)$ (Table 1). In the awake cohort, the mean score was $59.3 \pm$ 13.1 at baseline and $32.3 \pm 7.0$ postoperatively, a decrease of 27.0 points $(45.5 \%)$. This was not statistically different from the amount of score decrease in the asleep cohort ( $\mathrm{p}$ $=0.35$ ). The observed power was below the standard 0.80, with $0.25,0.07$, and 0.15 power to detect a statistically significant difference in the mean baseline scores, postoperative scores, and change in scores, respectively.

\section{Stereotactic Plan}

\section{Asleep Cohort}

In the asleep cohort, $53 \%$ of patients were male $(\mathrm{n}=$ 9/17) with a mean age of $67.1 \pm 11.4$ years. A total of 29 leads were placed: 16 on the left side and 13 on the right. There were no significant mean differences between the 
TABLE 1. Preoperative and postoperative Bain and Findley Tremor Activities of Daily Living Questionnaire scores

\begin{tabular}{lccc}
\hline \multirow{2}{*}{ Variable } & $\begin{array}{c}c \\
\text { Asleep DBS } \\
(n=11)\end{array}$ & $\begin{array}{c}\text { Awake DBS } \\
(n=18)\end{array}$ & p Value \\
\hline Baseline score & $66.0 \pm 13.3$ & $59.3 \pm 13.1$ & 0.20 \\
\hline Postop score & $33.9 \pm 13.1$ & $32.3 \pm 7.0$ & 0.66 \\
\hline Change in score & $-32.1 \pm 15.7$ & $-27.0 \pm 12.4$ & 0.35 \\
\hline
\end{tabular}

left- and right-side Euclidean and radial errors, or target measurements. Absolute values were used for X coordinate measurements. Means, standard deviations, and $p$ values are shown in Table 2. The mean intercommissural distance (AC-PC) was $25.5 \mathrm{~mm}$. The mean target location was $X=13.4 \mathrm{~mm}, Y=-6.2 \mathrm{~mm}$, and $\mathrm{Z}=0 \mathrm{~mm}$. The mean coronal angle was $19.6^{\circ}$, and mean sagittal angle was $58.1^{\circ}$.

Two patients in the asleep group undergoing pulse generator placement on the same day as DBS lead placement were excluded from case and operating room time calculations. The mean case time for the asleep cohort was 105 \pm 28 minutes (unilateral, $93.5 \pm 25.2$ minutes; bilateral, $109.5 \pm 29.3$ minutes), and mean operating room time was $202 \pm 50$ minutes (unilateral, $192.5 \pm 53.1$ minutes; bilateral, $205.6 \pm 51.2$ minutes) (Table 3).

\section{Awake Cohort}

In the awake cohort, $70 \%$ of patients were male $(\mathrm{n}=$ $28 / 40$ ) with a mean age of $66.3 \pm 13.6$ years. A total of 60 leads were placed within this cohort, 40 on the left and 20 on the right side. Similar to the asleep cohort, measurements did not vary significantly for left versus right side (Table 2). The mean intercommissural distance for all leads placed was $25.2 \mathrm{~mm}$. The mean target was $X=13.5$ $\mathrm{mm}, \mathrm{Y}=6.0 \mathrm{~mm}$, and $\mathrm{Z}=0 \mathrm{~mm}$. The mean coronal angle was $19.6^{\circ}$, and the mean sagittal angle was $57.4^{\circ}$. There was no statistically significant difference in plan coordinates between asleep and awake cohorts.

Six patients in the awake group undergoing pulse generator placement on the same day as the DBS procedure were excluded from case and operating room time calculations. The mean case time for the awake cohort was 101.5 \pm 46.6 minutes (unilateral, $84.1 \pm 43.0$ minutes; bilateral, $118.9 \pm 44.7$ minutes); the mean operating room time was $167.6 \pm 52.1$ minutes (unilateral, $146.4 \pm 50.9$ minutes; bilateral, $188.8 \pm 45.4$ minutes). There were no statistically significant differences in mean case times between the awake versus asleep cohorts for unilateral cases $(p=0.69)$ or bilateral cases $(p=0.55)$ or in operating room times for the unilateral cases $(\mathrm{p}=0.13)$ or bilateral cases $(\mathrm{p}=0.39)$ (Table 3). There was no significant difference in the average third ventricular width measured at the AC-PC plane for the asleep versus awake cohorts $(4.8 \pm 2.0$ vs $5.1 \pm 2.2$ $\mathrm{mm}$, respectively; $\mathrm{p}=0.5$ ).

\section{Accuracy of Lead Placement}

Based on iCT measurements, the average Euclidean error was higher for the awake versus asleep cohort (1.7 \pm 0.8 vs $1.2 \pm 0.4 \mathrm{~mm}$, respectively; $\mathrm{p}=0.01$ ), and the average radial error was higher for the awake versus the asleep cohort at the trend level $(1.3 \pm 0.8$ vs $0.9 \pm 0.5 \mathrm{~mm}$, respectively; $p=0.06$ ). In subanalysis, the mean Euclidean error on the left side was significantly higher for awake patients compared with asleep patients $(1.7 \pm 0.9$ vs $1.2 \pm 0.4$ $\mathrm{mm}$, respectively; $\mathrm{p}=0.01$ ), trending toward significance for the right side $(1.5 \pm 0.7$ vs $1.1 \pm 0.5 \mathrm{~mm}$, respectively; $\mathrm{p}=0.06$ ). Radial error, on average, was also higher in the awake cohort compared with asleep cohort for the left (1.5 \pm 0.9 vs $1.0 \pm 0.6 \mathrm{~mm}$, respectively; $\mathrm{p}=0.05$ ) and right sides $(1.1 \pm 0.6$ vs $0.8 \pm 0.5 \mathrm{~mm}$, respectively; $\mathrm{p}=0.10)$, both at the trend level.

There was no significant difference in the average number of brain penetrations for leads placed in awake $(1.1 \pm 0.3)$ versus asleep $(1.03 \pm 0.18)$ cohorts $(p=0.25)$. One asleep patient required 2 brain penetrations for 1 lead placement, and 4 awake patients (7 leads) required 2 brain penetrations each for lead placement. The number of penetrations was not significantly different between the left and right sides within the awake cohort $(\mathrm{p}=0.27)$. The asleep patient requiring 2 brain penetrations had bilateral leads placed in the standard fashion as detailed above. The iCT scan demonstrated the left-sided lead to be $0.7 \mathrm{~mm}$ medial to the target and the right-sided lead to be $2.3 \mathrm{~mm}$ medial to the target. Given that the right-sided lead was greater than $2 \mathrm{~mm}$ medial to the target, the lead was re-

TABLE 2. Target coordinates and lead error measurements for asleep and awake patient cohorts

\begin{tabular}{|c|c|c|c|c|c|c|c|c|}
\hline \multirow[b]{3}{*}{ Variable } & \multirow{2}{*}{\multicolumn{2}{|c|}{ Asleep Leads $(n=29)^{*}$}} & \multirow{2}{*}{\multicolumn{2}{|c|}{ Awake Leads $(n=60)^{*}$}} & \multicolumn{4}{|c|}{$p$ Value } \\
\hline & & & & & \multicolumn{2}{|c|}{ Lt vs Rt Side } & \multicolumn{2}{|c|}{ Awake vs Asleep } \\
\hline & Lt Side $(n=16)$ & Rt Side $(n=13)$ & Lt Side $(n=40)$ & Rt Side $(n=20)$ & Asleep & Awake & Lt Side & Rt Side \\
\hline AC-PC (mm) & $25.6 \pm 1.7$ & $25.4 \pm 1.6$ & $25.2 \pm 1.9$ & $25.2 \pm 1.8$ & 0.75 & $>0.99$ & 0.46 & 0.74 \\
\hline Target X (mm) & $13.5 \pm 0.9$ & $13.3 \pm 0.9$ & $13.6 \pm 0.8$ & $13.4 \pm 0.9$ & 0.61 & 0.37 & 0.61 & 0.77 \\
\hline Target Y (mm) & $-6.2 \pm 0.5$ & $-6.2 \pm 0.5$ & $-6.2 \pm 0.6$ & $-5.8 \pm 1.1$ & 0.81 & 0.11 & 0.70 & 0.18 \\
\hline Target Z (mm) & $0.0 \pm 0.0$ & $0.0 \pm 0.0$ & $0.0 \pm 0.0$ & $-0.1 \pm 0.4$ & 0.18 & 0.28 & 0.38 & 0.41 \\
\hline Parasagittal angle $\left(^{\circ}\right)$ & $58.4 \pm 4.7$ & $57.7 \pm 3.9$ & $57 \pm 8.0$ & $58.3 \pm 3.6$ & 0.67 & 0.91 & 0.88 & 0.68 \\
\hline Coronal angle $\left({ }^{\circ}\right)$ & $20.3 \pm 5.6$ & $18.7 \pm 4.8$ & $19.7 \pm 8.5$ & $19.3 \pm 4.0$ & 0.42 & 0.86 & 0.80 & 0.67 \\
\hline Euclidean error (mm) & $1.2 \pm 0.4$ & $1.1 \pm 0.5$ & $1.7 \pm 0.9$ & $1.5 \pm 0.7$ & 0.48 & 0.48 & 0.01 & 0.06 \\
\hline Radial error (mm) & $1.0 \pm 0.6$ & $0.8 \pm 0.5$ & $1.5 \pm 0.9$ & $1.1 \pm 0.6$ & 0.28 & 0.12 & 0.05 & 0.10 \\
\hline
\end{tabular}

* There were 17 patients in the asleep DBS group and 40 patients in the awake DBS group. Mean \pm SD are presented for target coordinates and error measurements. 
positioned, utilizing a 2-mm lateral offset. Postplacement iCT scanning demonstrated the new lead to be $0.6 \mathrm{~mm}$ lateral to the target. In the cohort of 4 awake patients requiring a second brain penetration, all offsets for the repositioning were anterior through a $2-\mathrm{mm}$ offset. In all cases, tremor arrest was obtained through the first pass, and the rationale for repositioning the lead was sustained paresthesias. Postoperative imaging revealed a final lead position averaging $0.27 \pm 0.59 \mathrm{~mm}$ anterior and $0.89 \pm$ $0.98 \mathrm{~mm}$ medial to the intended target.

There were no intraoperative or perioperative complications in either cohort. Intraoperative complications were defined as any unexpected intraoperative event requiring early termination of the surgery (e.g., seizure, uncontrolled hypertension), or any acute hemorrhage or stroke visualized on iCT. Perioperative complications included any postoperative seizure or new neurological deficit, hospital readmission, hardware malfunction, wound infection, or return to the operating room within 6 months of surgery.

Stimulator settings were available for 17 leads in 11 asleep patients. At 1-6 months postoperatively (mean 2.4 \pm 1.7 months), the mean voltage was $2.2 \pm 0.8 \mathrm{~V}$, the mean pulse width was $69.4 \pm 10.9 \mu \mathrm{sec}$, and the mean frequency was $160.0 \pm 26.3 \mathrm{~Hz}$. The most frequent active contact for the asleep cohort was 1 for left-sided leads and 8 for rightsided leads.

Settings were also available for 30 leads in 22 awake patients. At $1-9$ months postoperatively (mean $4.3 \pm 3.2$ months), the mean voltage was $2.1 \pm 1.2 \mathrm{~V}$, the mean pulse width was $74.0 \pm 17.3 \mu \mathrm{sec}$, and the mean frequency was $172.3 \pm 22.4 \mathrm{~Hz}$. The most frequent active contacts were 0 and 1 for left-sided leads and 10 for right-sided leads. There was no statistically significant difference in settings between asleep and awake cohorts. Significance values for comparisons between awake and asleep cohorts were $p=$ 0.66 for mean voltage, $\mathrm{p}=0.10$ for pulse width, $\mathrm{p}=0.27$ for frequency, and $\mathrm{p}=0.99$ and $\mathrm{p}=0.17$, respectively, for active-contact left and right sides.

\section{Discussion}

DBS has been an FDA-approved therapy for Parkinson's disease and essential tremor for over a decade. It is safe and effective; however, not all patients who could benefit from this procedure are able to undergo "awake" surgery. Many patients have medically prohibitive comorbidities, e.g., obstructive sleep apnea, claustrophobia, or severe hypertension. Even those who are medically quali- fied may choose not to undergo awake DBS. While many factors (e.g., necessity of permanent hardware implantation) may contribute to this decision, fear, anxiety, and the potential discomfort of not being under general anesthesia for a surgical procedure may play a significant role.

\section{Targeting}

Over the last few years, improved MRI resolution of nuclei targeted in Parkinson's disease and increased availability of intraoperative imaging have made verifying intraoperative stereotactic accuracy more feasible. Thus, performing DBS under general anesthesia has been considered. $8,19,20,24,32$ Identifying the optimal DBS target within the subthalamic nucleus or globus pallidus pars interna on MRI remains controversial. However, this so-called "direct targeting" has not been possible with VIM. Although some have reported being able to visualize the VIM using available MR images, the VIM $^{31}$ is generally targeted via indirect or atlas-based methods. Variability in consensus coordinates for the VIM presents the primary challenge to performing asleep DBS for essential tremor. ${ }^{14,27}$ Osenbach and Burchiel suggested targeting the VIM with the following coordinates: $\mathrm{X}, 13-15 \mathrm{~mm}$ lateral to the intercommissural line (IC) (11 $\mathrm{mm}$ lateral to the third ventricular wall if the ventricle width exceeds $6 \mathrm{~mm}$ ); Y, $25 \%$ of the IC anterior to the posterior commissure; and $\mathrm{Z}$, in the same axial image as the IC..$^{23}$ Other groups disagree and have demonstrated that differences in individual patient anatomy decrease the reliability of atlas-based targets. ${ }^{7,14,15}$

We acknowledge this lack of precise definition in our VIM targeting criteria, while postulating that accurate lead placement to a target selected using indirect methods will nonetheless result in expected tremor control. This is the first report of performing VIM DBS for essential tremor under general anesthesia without intraoperative test stimulation. In this analysis of our initial experience with asleep VIM DBS, we found that the procedure could be performed safely with submillimeter stereotactic accuracy (radial error $0.9 \mathrm{~mm}$ ) and excellent clinical efficacy (Bain and Findley Tremor ADL score improvement 48.6\%). The degree of improvement seen was not statistically different from that seen in our patients undergoing traditional awake DBS with intraoperative test stimulation. All patients who responded to the tremor ADL questionnaire demonstrated a decrease in tremor ADL scores postoperatively, indicating an increased ability to independently perform ADLs affected by tremor and a decreased degree of tremor-related disability.

TABLE 3. Case and operating room time for DBS procedures

\begin{tabular}{|c|c|c|c|c|c|}
\hline \multirow[b]{2}{*}{ Variable } & \multicolumn{2}{|c|}{ Asleep } & \multicolumn{2}{|c|}{ Awake } & \multirow[b]{2}{*}{ p Value } \\
\hline & Mean \pm SD & No. of Leads & Mean \pm SD & No. of Leads & \\
\hline \multicolumn{6}{|c|}{ Case time (mins) } \\
\hline Unilateral & $93.5 \pm 25.2$ & 4 & $84.1 \pm 43.0$ & 15 & 0.69 \\
\hline Bilateral & $109.5 \pm 29.3$ & 11 & $118.9 \pm 44.7$ & 15 & 0.55 \\
\hline \multicolumn{6}{|c|}{ OR time (mins) } \\
\hline Unilateral & $192.5 \pm 53.1$ & 4 & $146.4 \pm 50.9$ & 15 & 0.13 \\
\hline Bilateral & $205.6 \pm 51.2$ & 11 & $188.8 \pm 45.4$ & 15 & 0.39 \\
\hline
\end{tabular}

$\mathrm{OR}=$ operating room . 


\section{Stereotactic Accuracy and Lead Repositioning}

Our accuracy is comparable to traditional DBS placement without general anesthesia, as well as to that in other recently published series of image-guided DBS under general anesthesia (Burchiel et al., ${ }^{8} 1.2$-mm radial error using iCT; Starr et al., ${ }^{32} 1.2-\mathrm{mm}$ radial error using iMRI). The increased stereotactic error $(\mathrm{p}=0.01)$ and number of lead repositionings $(p=0.25)$ in the awake group may be due to a variety of factors.

\section{Lead Repositioning}

First, all of our lead repositionings in the awake cohort were performed for low thresholds for sensory paresthesias, not for absence of clinical benefit or capsular side effects. However, the clinical relevance of intraoperative paresthesias occurring immediately after lead placement is unclear, and it is possible that, if those leads had not been repositioned, clinical benefit and side effect profiles may have nonetheless been acceptable. Second, on postoperative CT, the 2-mm anterior offset had the effect of positioning the lead an average of $0.27 \mathrm{~mm}$ anterior to the intended target, i.e., less distance than expected. In other words, the initial trajectory may have had a posterior stereotactic error, and this was brought to our attention and corrected for with intraoperative test stimulation. This suggests that the offset possibly corrected for stereotactic error rather than targeting error. In an analogous situation in asleep surgery, it is possible that intraoperative imaging would have similarly demonstrated a posteriorly placed lead, and repositioning might have been done based on the stereotactic error detected rather than test stimulation.

\section{Stereotactic Error}

While we did not perform intraoperative fluoroscopy to detect whether we were targeting the center of the frame, we hypothesize that the error in awake surgery may have arisen from brain shift from moving the patient from a $\mathrm{HOB}$ at $0^{\circ}$ for $\mathrm{CT}$ and MRI to $\mathrm{HOB}$ at $30^{\circ}$ for awake surgery. Alternatively, error could also arise from possible micromovements of the frame during transport to and from the radiology department for the CT scan, which was the workflow for the first 16 awake patients. With the asleep cohort, once the patient is positioned with the HOB at $0^{\circ}$ with the frame on, there is no change in positioning from the time of first CT scan to the time of skin closure. For both awake and asleep surgery, all scans are now performed in the operating room without moving the patient from the operating room table. The one main technical difference between awake and asleep surgery is that awake surgery is still performed with $\mathrm{HOB}$ at $30^{\circ}$. Since we have minimized differences in surgical technique between awake and asleep surgery, the differences in stereotactic error have also decreased.

\section{Case and Operating Room Time}

Currently there are no significant differences in case or operating room times for asleep VIM DBS compared with the traditional awake method. However, the ability to compare the actual case and operating room times for both cohorts is confounded by several factors. Confounding factors that would reduce the time for awake surgery include 1) frame placement before coming into the operating room
(6 patients), 2) registration CT taking place before coming into the operating room (16 patients), and 3) postlead placement $\mathrm{CT}$ occurring after leaving the operating room (12 patients). Confounding factors that would increase the time measured for asleep surgery include 1) use of NexFrame in 5 patients (our recent multivariate analysis demonstrated that Nexframe was associated with significantly increased case and operating room times compared with the Leksell frame by 0.5 and 1.1 hours, respectively ${ }^{18}$ ); 2) all CT scans taking place in the operating room; and 3) time required to induce general anesthesia.

Since January 2013, the protocols have been standardized (frame placement and CT scanning occur in the operating room for both cohorts), and our next prospective analysis will reflect more accurate comparisons of case and operating room times.

\section{Limitations}

There are several limitations to our current study, the most significant being its retrospective and nonrandomized nature. Comparing functional outcomes between 2 different surgical techniques for the same procedure in a nonrandomized fashion introduces inherent weaknesses. For example, patients requesting one method over the other may have expectations preoperatively that could bias responses to the ADL questionnaires. Given that our data were collected retrospectively, a number of patients did not respond to the ADL questionnaire (35.3\% of asleep patients and $52 \%$ of awake patients).

In addition, the length of follow-up was inconsistent and incomplete. For instance, there was a wide range of questionnaire follow-up times in the asleep group (4-29 months postoperatively) and awake group (4-31 months postoperatively). Nonstandardized follow-up times could affect data accuracy. Furthermore, recall bias is introduced from patients completing ADL questionnaires postoperatively for both pre- and postoperative time points. All patients, regardless of response to questionnaire, were seen in clinic at both 2 weeks and 3 months postoperatively. At the 3-month follow-up, patients who had not responded to the questionnaire did report significant tremor control and ADL independence improvement.

An additional limitation of this study is the lack of an objective tremor measure documented at baseline and postoperatively (e.g., the Fahn-Talosa-Marin tremor scale or other tremor rating scale). ${ }^{9,10,17}$ It is only inferred that the clinical improvement seen in decreased ADL disability scores results from reduced tremor severity.

\section{Future Directions}

To address the aforementioned limitations, we have since modified our clinical protocol to include a standardized preoperative and 3-month postoperative assessment for all patients with essential tremor who undergo VIM DBS placement under general anesthesia (clinicaltrials. gov identifier: NCT01909531). Data points collected include the Fahn-Talosa-Marin tremor scale, Bain and Findley Tremor ADL questionnaire, Quality of life in Essential Tremor (QUEST), ${ }^{33}$ and 2 iPhone accelerometer applications measuring tremor frequency and intensity (Lift Pulse and Parkinson Meter). All asleep and awake VIM DBS 
patients currently undergo this protocol, and a randomized study comparing outcomes between awake and asleep VIM DBS patients has been approved by our institutional review board and is currently underway (clinicaltrials.gov identifier: NCT02418858).

\section{Conclusions}

VIM DBS for essential tremor can be performed safely and effectively under general anesthesia without test stimulation, with excellent patient outcomes and satisfaction. We are currently validating the results of this initial experience in a prospective study.

\section{Acknowledgments} tion.

Funding was provided by the Barrow Center for Neuromodula-

\section{References}

1. Alexander E III, Kooy HM, van Herk M, Schwartz M, Barnes PD, Tarbell N, et al: Magnetic resonance image-directed stereotactic neurosurgery: use of image fusion with computerized tomography to enhance spatial accuracy. J Neurosurg 83:271-276, 1995

2. Alterman RL, Weisz D: Microelectrode recording during deep brain stimulation and ablative procedures. Mov Disord 27:1347-1349, 2012

3. Alusi SH, Worthington J, Glickman S, Findley LJ, Bain PG: Evaluation of three different ways of assessing tremor in multiple sclerosis. J Neurol Neurosurg Psychiatry 68:756-760, 2000

4. Anthofer J, Steib K, Fellner C, Lange M, Brawanski A, Schlaier J: The variability of atlas-based targets in relation to surrounding major fibre tracts in thalamic deep brain stimulation. Acta Neurochir (Wien) 156:1497-1504, 2014

5. Bain PG, Findley LJ, Atchison P, Behari M, Vidailhet M, Gresty M, et al: Assessing tremor severity. J Neurol Neurosurg Psychiatry 56:868-873, 1993

6. Benabid AL, Chabardes S, Mitrofanis J, Pollak P: Deep brain stimulation of the subthalamic nucleus for the treatment of Parkinson's disease. Lancet Neurol 8:67-81, 2009

7. Brierley JB, Beck E: The significance in human stereotactic brain surgery of individual variation in the diencephalon and globus pallidus. J Neurol Neurosurg Psychiatry 22:287298, 1959

8. Burchiel KJ, McCartney S, Lee A, Raslan AM: Accuracy of deep brain stimulation electrode placement using intraoperative computed tomography without microelectrode recording. J Neurosurg 119:301-306, 2013

9. Fields JA, Tröster AI, Woods SP, Higginson CI, Wilkinson SB, Lyons KE, et al: Neuropsychological and quality of life outcomes 12 months after unilateral thalamic stimulation for essential tremor. J Neurol Neurosurg Psychiatry 74:305311,2003

10. Flora ED, Perera CL, Cameron AL, Maddern GJ: Deep brain stimulation for essential tremor: a systematic review. Mov Disord 25:1550-1559, 2010

11. Foltynie T, Zrinzo L, Martinez-Torres I, Tripoliti E, Petersen E, Holl E, et al: MRI-guided STN DBS in Parkinson's disease without microelectrode recording: efficacy and safety. J Neurol Neurosurg Psychiatry 82:358-363, 2011

12. Gross RE, McDougal ME: Technological advances in the surgical treatment of movement disorders. Curr Neurol Neurosci Rep 13:371, 2013

13. Harries AM, Kausar J, Roberts SA, Mocroft AP, Hodson JA, Pall HS, et al: Deep brain stimulation of the subthalamic nucleus for advanced Parkinson disease using general anesthesia: long-term results. J Neurosurg 116:107-113, 2012

14. Hirabayashi H, Tengvar M, Hariz MI: Stereotactic imaging of the pallidal target. Mov Disord 17 (Suppl 3):S130-S134, 2002

15. Kelly PJ, Derome P, Guiot G: Thalamic spatial variability and the surgical results of lesions placed with neurophysiologic control. Surg Neurol 9:307-315, 1978

16. Kooy HM, van Herk M, Barnes PD, Alexander E III, Dunbar $\mathrm{SF}$, Tarbell NJ, et al: Image fusion for stereotactic radiotherapy and radiosurgery treatment planning. Int J Radiat Oncol Biol Phys 28:1229-1234, 1994

17. Lee JY, Kondziolka D: Thalamic deep brain stimulation for management of essential tremor. J Neurosurg 103:400-403, 2005

18. Mirzadeh Z, Chapple K, Lambert M, Dhall R, Ponce FA: Stereotactic technique determines accuracy and efficiency in "asleep" DBS. Sterotact Funct Neurosurg 92 (Suppl 1):36, 2014 (Abstract)

19. Mirzadeh Z, Chapple K, Lambert M, Dhall R, Ponce FA: Validation of CT-MRI fusion for intraoperative assessment of stereotactic accuracy in DBS surgery. Mov Disord 29:17881795,2014

20. Mirzadeh Z, Chapple K, Lambert M, Evidente VG, Mahant P, Ospina MC, et al: Parkinson's disease outcomes after intraoperative CT-guided "asleep" deep brain stimulation in the globus pallidus internus. J Neurosurg [epub ahead of print October 9, 2015; DOI: 10.3171/2015.4.JNS1550]

21. Montgomery EB Jr: Microelectrode targeting of the subthalamic nucleus for deep brain stimulation surgery. Mov Disord $27: 1387-1391,2012$

22. Nakajima T, Zrinzo L, Foltynie T, Olmos IA, Taylor C, Hariz MI, et al: MRI-guided subthalamic nucleus deep brain stimulation without microelectrode recording: can we dispense with surgery under local anaesthesia? Stereotact Funct Neurosurg 89:318-325, 2011

23. Osenbach R, Burchiel K: Thalamotomy: indications, techniques, and results, in Germano IM (ed): Neurosurgical Treatment of Movement Disorders. Park Ridge, IL: American Association of Neurological Surgeons, 1998

24. Ostrem JL, Galifianakis NB, Markun LC, Grace JK, Martin AJ, Starr PA, et al: Clinical outcomes of PD patients having bilateral STN DBS using high-field interventional MR-imaging for lead placement. Clin Neurol Neurosurg 115:708-712, 2013

25. Papanastassiou V, Rowe J, Scott R, Silburn P, Davies L, Aziz T: Use of the Radionics Image Fusion ${ }^{\mathrm{TM}}$ and Stereoplan ${ }^{\mathrm{TM}}$ programs for target localization in functional neurosurgery. $\mathbf{J}$ Clin Neurosci 5:28-32, 1998

26. Pezeshkian P, DeSalles AA, Gorgulho A, Behnke E, McArthur D, Bari A: Accuracy of frame-based stereotactic magnetic resonance imaging vs frame-based stereotactic head computed tomography fused with recent magnetic resonance imaging for postimplantation deep brain stimulator lead localization. Neurosurgery 69:1299-1306, 2011

27. Sandvik U, Koskinen LO, Lundquist A, Blomstedt P: Thalamic and subthalamic deep brain stimulation for essential tremor: where is the optimal target? Neurosurgery 70:840846,2012

28. Schaltenbrand G, Wahren W, Hassler RG: Atlas for Stereotaxy of the Human Brain, ed 2. Stuttgart: Thieme, 1977

29. Schlaier J, Anthofer J, Steib K, Fellner C, Rothenfusser E, Brawanski A, et al: Deep brain stimulation for essential tremor: targeting the dentato-rubro-thalamic tract? Neuromodulation 18:105-112, 2015

30. Shin M, Penholate MF, Lefaucheur JP, Gurruchaga JM, Brugieres P, Nguyen JP: Assessing accuracy of the magnetic resonance imaging-computed tomography fusion images to evaluate the electrode positions in subthalamic nucleus after deep-brain stimulation. Neurosurgery 66:1193-1202, 2010 
31. Spiegelmann R, Nissim O, Daniels D, Ocherashvilli A, Mardor Y: Stereotactic targeting of the ventrointermediate nucleus of the thalamus by direct visualization with highfield MRI. Stereotact Funct Neurosurg 84:19-23, 2006

32. Starr PA, Martin AJ, Ostrem JL, Talke P, Levesque N, Larson PS: Subthalamic nucleus deep brain stimulator placement using high-field interventional magnetic resonance imaging and a skull-mounted aiming device: technique and application accuracy. J Neurosurg 112:479-490, 2010

33. Tröster AI, Pahwa R, Fields JA, Tanner CM, Lyons KE: Quality of life in Essential Tremor Questionnaire (QUEST): development and initial validation. Parkinsonism Relat Disord 11:367-373, 2005

\section{Disclosures}

Dr. Ponce is a consultant for Medtronic.

\section{Author Contributions}

Conception and design: Ponce, Chen. Acquisition of data: Ponce, Chen, Lambert. Analysis and interpretation of data: Ponce, Chen, Mirzadeh, Chapple. Drafting the article: Chen, Mirzadeh. Critically revising the article: Ponce, Dhall. Reviewed submitted version of manuscript: Ponce. Approved the final version of the manuscript on behalf of all authors: Ponce. Statistical analysis: Chen, Chapple. Administrative/technical/material support: Lambert. Study supervision: Ponce.

\section{Correspondence}

Francisco A. Ponce, c/o Neuroscience Publications, Barrow Neurological Institute, St. Joseph's Hospital and Medical Center, 350 W. Thomas Rd., Phoenix, AZ 85013.email: neuropub@ dignityhealth.org. 